• 研究报告・

\title{
基于基因组SNPs的南极恩克斯堡岛阿德利 企鹅繁殖种群的遗传结构
}

\author{
张 剑 董 路 张雁云* \\ (生物多样性与生态工程教育部重点实验室, 北京师范大学生命科学学院, 北京 100875)
}

\begin{abstract}
摘要: 我国提议在南极恩克斯堡岛新站址北侧 $3 \mathrm{~km}$ 的阿德利企鹅(Pygoscelis adeliae)聚集繁殖地建立南极特别保 护区, 对保护区边界的划分, 各国尚存争议, 尤其是对南湾(South Bay)的繁殖小种群是否具有遗传独特性, 是否 应将其纳入保护区是重点关注的问题。本研究采集了恩克斯堡岛海景湾(Seaview Bay)和南湾的阿德利企我样品, 通过全基因组重测序和种群基因组学方法, 分析了恩克斯堡岛不同区域的种群遗传结构。发现恩克斯堡岛海景湾 与南湾阿德利企鹅没有显著的遗传分化, 南湾阿德利企鹅不是独特的小种群; 海景湾高海拔区域个体与低海拔区 域个体之间也没有显著的遗传差异, 推测该区域阿德利企鹅繁殖群体的分布格局与冰川堆积形成的阶地不具有显 著相关性。本工作为恩克斯堡岛保护区和罗斯海新站建设提供了重要科技支撑。
\end{abstract}

关键词：恩克斯堡岛；阿德利企我；遗传结构；南极特别保护区; SNPs

\section{Population genetic structure of Adélie penguins (Pygoscelis adeliae) from Inexpressible Island, Antarctica, using SNP markers}

Jian Zhang, Lu Dong, Yanyun Zhang*

Ministry of Education Key Laboratory for Biodiversity and Ecological Engineering; College of Life Sciences, Beijing Normal University, Beijing 100875

Abstract: China has proposed to establish an Antarctic Specially Protected Area (ASPA) to protect the
Adélie penguin, Pygoscelis adeliae, colonies on Inexpressible Island. However, the boundary of the proposed
ASPA is disputed mainly by whether the South Bay population is a distinct genetic population in need of
protection. We collected samples of Adélie penguins from the South Bay and neighboring Seaview Bay on
Inexpressible Island and identified SNPs via Illumina sequencing for population structure analysis. Our
results indicated that there was no significant genetic difference between the South Bay colonies and the
Seaview Bay colonies, or between the higher and lower elevational colonies in the Seaview Bay. Thus, the
breeding populations of Adélie penguin on the island could be recognized as a single conservation unit. The
currently proposed boundary of the ASPA, which covers the majority of breeding populations in Seaview
Bay, would be sufficient to protect the genetic diversity of Adélie penguins in Inexpressible Island.

Key words: Inexpressible Island; Pygoscelis adeliae; population structure; Antarctic Specially Protected Area; SNPs

理解种群遗传结构和形成机制对于物种保护 非常重要(Manel et al, 2003), 当物种内种群间具有 较高的遗传分化且扩散受限制时, 繁殖群体应对不 确定的局部环境变化的能力会下降(Walther et al, 2002)。划定繁殖群体精确的地理界线能够更准确地
对种群数量动态进行监测, 从而提出更有效的管理 计划(Palsboll et al, 2007; Funk et al, 2012)。

基于种群遗传学方法划分繁殖种群并评估种 群连续性是制定有效保护策略的重要依据(Funk et al, 2012)。阿德利企我(Pygoscelis adeliae) 是南极陆

收稿日期: 2019-08-16; 接受日期: 2019-11-20

基金项目：南极恩克斯堡岛企鹅保护区项目(23020005)和南大洋鸟类多样性和分布格局调查项目(JDXT2019-02)

* 通讯作者 Author for correspondence. E-mail: zhangyy@bnu.edu.cn 
地动物的代表类群，约占南极鸟类生物量的 $90 \%$ (Croxall \& Prince, 1979; Borowicz et al, 2018)。阿德 利企我的繁殖地点集中在南极大陆无冰的沿海岸 区域, 常形成高密度的繁殖地, 目前最大的繁殖地 约有75万个繁殖对(Borowicz et al, 2018)。冬季在环 南极大陆的海冰上生活, 夏季初期到达南极大陆沿 海岸的无冰区域结成繁殖对, 个体表现出强烈的归 巢行为(Ainley et al, 1983), 这种行为可能是一种隔 离机制, 对基因流形成阻碍。利用线粒体DNA以及 少量核基因对西南极大陆阿德利企我的研究(Gorman et al, 2017), 以及利用单核苷酸多态性位点 (single nucleotide polymorphisms, SNPs)对泛南极区 域的研究(Clucas et al, 2018), 尚未发现各繁殖群体 间存在显著的遗传分化。然而这些研究都缺少罗斯 海地区阿德利企鹅的样本。作为冰川时代阿德利企 我的重要避难所, 罗斯海区域的阿德利企我种群可 能具有独特的遗传特征(Ritchie et al, 2004)。Shepherd等(2005)利用微卫星分子标记对恩克斯堡岛

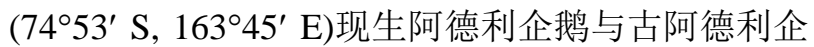
我的比较研究发现, 现生种群与古种群之间存在明 显的遗传分化, 但未对现生各繁殖种群之间的遗传 分化程度进行分析。

我国拟在南极罗斯海地区的恩克斯堡岛建立 第五个南极科学考察站。出于环境保护、科学研究 和发展战略的需要, 目前由中国牵头, 意大利和韩 国参加, 在恩克斯堡岛北部、距离站址 $3 \mathrm{~km}$ 处建立 南极特别保护区。在该区域共有阿德利企鹅 25,013 个繁殖对(2018年1月韩国考察队调查数据, Hosung Chung, 个人通讯), 由花岗岩形成的陆岬将该区域 划分为海景湾和南湾两个繁殖区域, 其中海景湾区 域分布有24,940个繁殖对, 南湾区域分布有73个繁 殖对。对挖掘出来的恩克斯堡岛的古阿德利企我骨 骼、蛋壳、粪便, 通过碳稳定同位素进行年代判定, 发现阿德利企鹅利用海景湾与南湾持续作为繁殖 地的历史超过7,000年(Baroni \& Orombelli, 1991), 也是罗斯海地区全新世以来持续繁殖时间最长的 种群(Baroni \& Hall, 2004; Emslie et al, 2007; Lorenzini et al, 2010)。保护区内阿德利企我繁殖群自 1963 年就有监测记录(Stonehouse, 1969), 是迄今罗斯海 地区阿德利企我最早的研究记录(Woehler \& Croxall, 1997), 并一直持续到现在(Lyver et al, 2014),
对于研究气候变化对种群动态的影响有重要的价值。

阿德利企我具有较强的归巢行为, 基于少量个 体的标记观测结果发现，罗斯海地区的阿德利企鹅 的归巢行为会受到冰山位置变动的影响(Shepherd et al, 2005), 但由于遗传数据的缺乏, 也还无法评 估归巢行为的波动是否会对这一地区不同繁殖群 体之间的基因流造成影响，因此目前难以确定是把 恩克斯堡岛的阿德利企鹅划分为一个大的保护单 元还是几个小的保护单元。此外, 海景湾繁殖地存 在常年积雪的带状地区(海拔20 m, 图1), 而阿德利 企鹅仅在裸露的地面繁殖, 雪线对该区域阿德利企 我鸟繁殖群的隔离是否导致遗传分化，也是划分保护 单元的重要影响因素。

中国主持完成的设立保护区的预评估报告已 经在第41次南极条约体系协商会议上得到通过, 并 同意由中方牵头撰写保护区管理计划草案，该草案 已经过美国、意大利、新西兰、韩国和德国科学家 的多轮讨论，但大家在边界确定上还存在分歧。南 湾的阿德利企鹅种群是否为特殊的小种群, 这涉及 到与繁殖地相接海域(也是我国科考船在科考站后 勤保障中可能停靠的区域)的保护范围。加强对恩克 斯堡岛阿德利企鹅的研究和保护, 不仅可以提升我 国对南极鸟类研究的水平，也可以体现我国环境保 护和参与全球环境治理的理念和行动。我们采集了 恩克斯堡岛阿德利企鹅海景湾和南湾两个主要繁 殖区域的样品，利用基因组重测序的方法笁选单核 苷酸多态性位点进行遗传多样性分析，以检测不同 地点的群体是否具有显著的遗传分化, 明晰种群结 构, 从而划定重点保护种群, 为制订更为有效的保 护计划提供信息，同时也为保护区边界的划定提供 理论依据。

\section{材料与方法}

\section{1 研究区域}

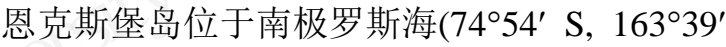
E), 阿德利企我繁殖地集中于恩克斯堡岛北部的海 景湾和南湾区域。企鹅的栖息地沿冰川漂砾堆积的 冰碛陇，形成不同高度的14级阶地地貌，区内海滩 在全新世抬升高度达 30 m (Baroni \& Orombelli, 1991), 冰碛陇南北走向, 西高东低, 总体形成西北 突出的弧形，企鹅的巢见于不同级阶地中。 


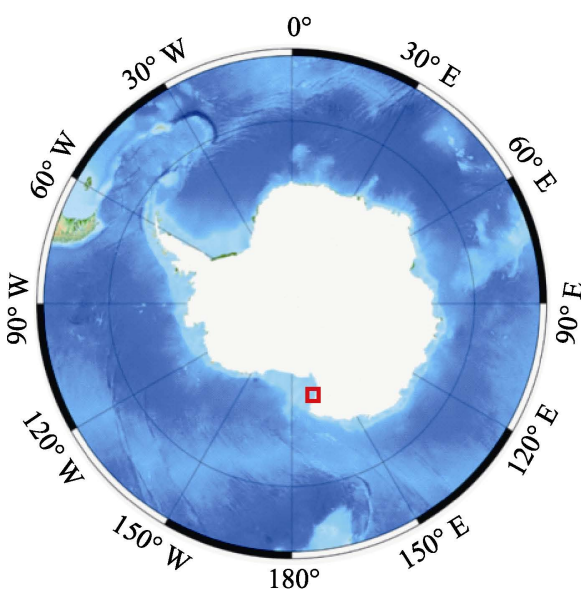

A
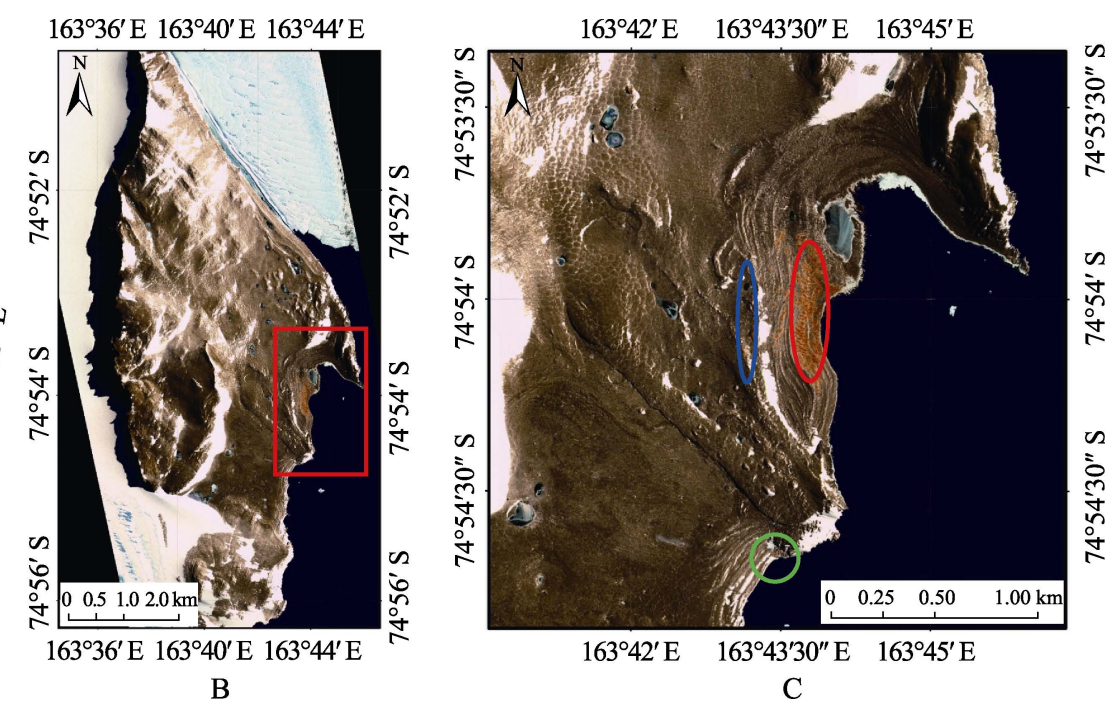

图1 阿德利企鹅采样区地图。A: 南极地图, 红框示恩克斯堡岛的位置; B: 恩克斯堡岛地图, 红框示拟建保护区的位置; C: 采样区域, 蓝色和红色圆圈分别为海景湾高海拔和低海拔采样区, 绿色圆圈为南湾采样区。

Fig. 1 Sampling sites of Adélie penguins (Pygoscelis adeliae) in Inexpressible Island. (A) Map of Antarctic (Red rectangle: Inexpressible Island). (B) Map of Inexpressible Island (Red rectangle: the proposed Antarctic Specially Protected Area). (C) Sampling areas in Inexpressible Island. Blue circle, high altitude area in Seaview Bay; red circle, low altitude area in Seaview Bay; green circle, South Bay.

区内气候条件严酷，依据1988-2012年气象资 料, 年平均气温为 $-18.5^{\circ} \mathrm{C}$, 每年低于 $-40^{\circ} \mathrm{C}$ 的天数 不超过 $0.1 \%$, 年均风速 $12.0 \mathrm{~m} / \mathrm{s}$, 日均最大风速为 $34.2 \mathrm{~m} / \mathrm{s}$, 最大瞬时风速 $45 \mathrm{~m} / \mathrm{s}$ 。在持续的强下降风 及Drygalski冰舌对浮冰阻隔的作用下, 当周围其他 水域都覆盖固定冰或浮冰的时候, 在海景湾和南湾 附近的特拉诺瓦湾形成了面积约为 $1,300 \mathrm{~km}^{2}$ 的巨 大冰间湖(Bromwich \& Kurtz, 1984), 为阿德利企我鸟 繁殖期受食育雉提供了条件, 这也可能是这些阿德利 企鹅长期在此繁衍的主要原因(Emslie et al, 2007)。

\section{2 样品采集与基因组DNA提取}

2018年1月参加我国第35次南极科学考察期间, 我们在恩克斯堡岛共采集自然死亡的阿德利企我鸟 样品147个, 采集样点见图1, 其中在南湾区域采集 样品15个, 在海景湾高海拔区域(海拔20-58 m) 采 集样品44个, 低海拔区域(海拔1-20 m)采集样品88 个。样品置于无水乙醇 $-20^{\circ} \mathrm{C}$ 保存。

使用TIANamp Genomic DNA Kit血液/组织/细 胞基因组DNA提取试剂盒(离心柱型)(天根, 北京) 提取基因组DNA。使用Qubit 3.0苂光定量仪测量 DNA浓度, 并利用琼脂糖凝胶电泳检测基因组 DNA的完整度。将浓度较高且基因组DNA比较完整 的86个样品进行全基因组重测序分析。首先通过 Covaris系统将基因组随机打断成长度为350 bp左右
的片段, 采用TruSeq Library Construction Kit (Illumina, 美国)进行建库, 在Illumina HiSeq平台以 PE 2×150 (Paired-End 150 bp)模式进行测序。

\section{3 数据分析}

\subsection{1 原始测序数据过滤和比对}

将原始测序序列(raw reads)进行过滤, 去除接 头和低质量的reads, 得到clean reads, 进行后续分 析。采用阿德利企鹅De novo基因组作为参考基因组 (Zhang et al, 2014), 应用BWA (Li \& Durbin, 2010) 将重测序结果比对至参考基因组。

\subsubsection{SNPs检测及篮选}

利用 Sentieon DNAseq 软件包 v.201711.05 (Freed et al, 2017)获取SNPs位点(emit_conf $=10$, call_conf = 30)。应用Perl v5.10.1编写脚本, 保留在 个体平均测序深度的 $1 / 3$ 至 2 倍的SNPs位点, 应用卡 方检验基于测序深度对等位基因频率进行校正 (Nielsen et al, 2011), 过滤单一序列标签(read)形成 的多态性位点(singleton)及存在缺失数据的位点。应 用PopLDdecay 3.31 (Zhang et al, 2019)进行连锁不 平衡(LD)分析, 选用间隔 $10 \mathrm{~kb}$ 以上的位点, 以确保 获得不连锁的独立SNPs位点。通过以上篮选获得的 SNPs位点用于后续的种群遗传结构分析。

\subsection{3 遗传多样性分析}

应用VCFtools 0.1.15 (Danecek et al, 2011)分别 
对海景湾个体和南湾个体的全部SNPs位点进行分 析, 计算 $\pi$ 值。

\subsection{4 种群结构分析}

应用基于最大似然法的 ADMIXTURE 1.3 .0 (Alexander et al, 2009)推算种群遗传结构, $\mathrm{K}$ 取值为 1-10, 根据交叉验证(cross-validation, CV)错误值判 断种群分组情况(K值), CV 值最小时所对应的 K值 为最佳分组数目。进而在指定个体地理信息的基础 上 $(\mathrm{K}=2$ : 海景湾区域与南湾区域; $\mathrm{K}=3$ : 海景湾 高海拔区域、海景湾低海拔区域和南湾区域), 进行 个体的种群归属(population assignment)分析, 如果 同一个地理种群的个体未形成独特的遗传单元(具 有共同祖先来源), 且不同地理种群的个体具有相 同的遗传来源, 则表明地理种群之间不存在显著的 遗传分化。利用基于贝叶斯算法的fastSTRUCTURE 1.0 (Raj et al, 2014)分析种群遗传结构, K值设为 1-10, seed 值设为 100, 并应用 SNPRelate 1.18 .1 (Zheng et al, 2012)进行主成分分析(principal component analysis, PCA), 评估种群分化程度。对亲缘 关系较近的个体仅保留其中一只纳入数据分析 (见 1.3.5节)。

\subsection{5 距离隔离效应分析}

利用PGDSpider 2.1.1.5 (Lischer \& Excoffier, 2012)进行数据格式转换。随机选择 8,000 个SNPs位 点, 利用GenALEx 6.51b2 (Peakall \& Smouse, 2012) 计算个体间的亲缘系数, 根据Ritland's (1996)算法 得到RI值, 对亲缘关系较近(RI >0.3)的个体保留其 中一只, 纳入后续分析。结合采样点的地理坐标进 行Mantel检验, 以检验是否存在伴随地理距离增加 而遗传分化程度增加的距离隔离效应(isolation by distance, IBD)

\section{2 结果}

\section{1 遗传多样性}

测序个体中共有 72 只阿德利企鹅与参考基因 组的比对率超过 $95 \%$ (海景湾高海拔区域25只 低海 拔区域39只; 南湾区域8只), 平均测序深度为 15.63 倍。其余样品可能存在来源于环境中的DNA污染, 导致比对率较低 $(<90 \%)$ 。基于亲缘关系分析排除 10 个亲缘关系较近的个体, 应用 62 个个体进行后续分 析。基于种群数据获取SNPs位点50,598,094个, 篮 选后用于数据分析的SNPs位点共 110,451 个。
海景湾区域所有个体的遗传多样性 $(\pi)$ 为 $0.153 \%$, 其中高海拔为 $0.152 \%$ ，低海拔区域为 $0.153 \%$ 。南湾 区域所有个体的遗传多样性 $(\pi)$ 为 $0.153 \%$ 。

\section{2 种群遗传结构}

基于最大似然法估计的地理种群最佳分组数 目为 1, 即没有显著的遗传分化。在根据样品采集地 进行分组估算个体的祖先来源时，不同种群的个体 未形成明显的分组(图2), 表明各种群之间的基因流 频繁，不存在显著的遗传分化。应用贝叶斯法估计 的最佳分组数目同样为 1 。主成分分析表明，虽然个 体间存在一定程度的分化(主成分 1 ), 但是海景湾高
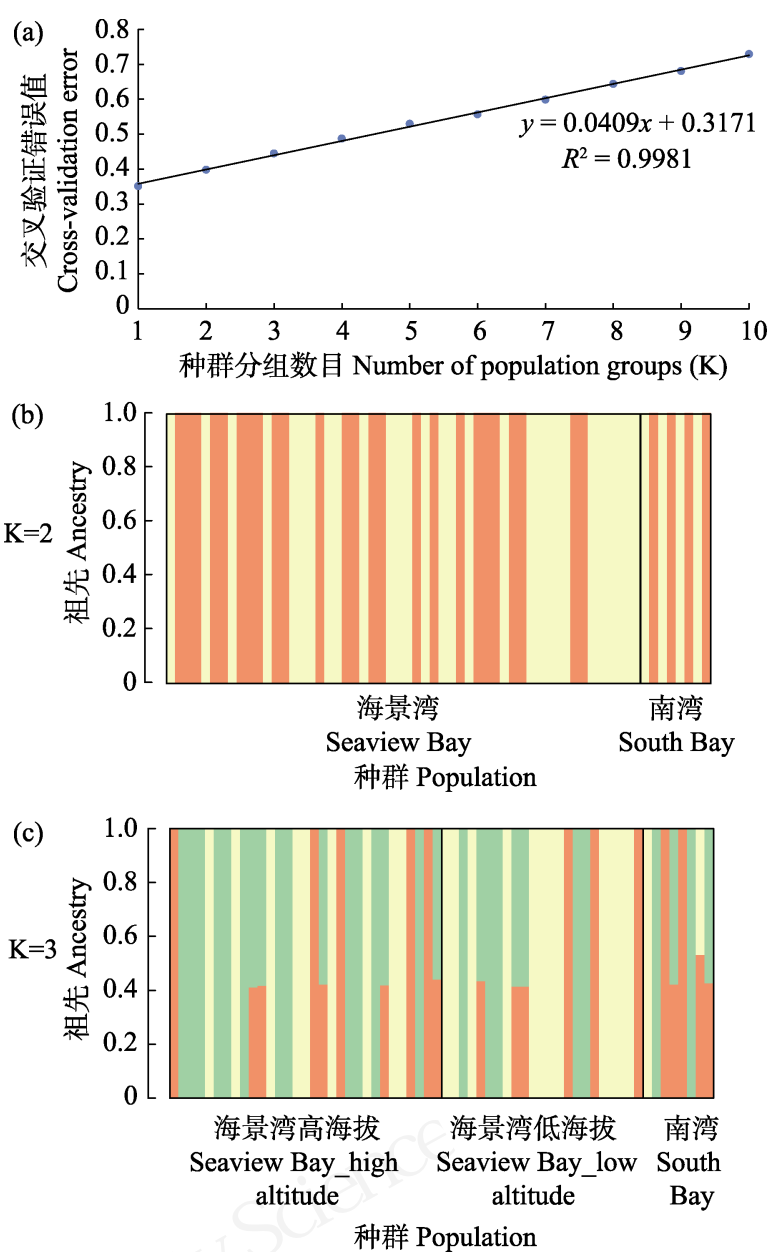

图2 基于110,451个SNPs利用最大似然法构建的恩克斯堡 岛阿德利企我的种群遗传结构。(a)交叉验证错误率分布; (b) $K=2 ;(c) K=3$ 。图中每一坚条为 1 个个体, 用颜色表示每个 个体基因组中不同祖先所占的比例。

Fig. 2 Based on 110,451 SNPs, the population genetic structure of Adélie penguins was constructed by maximum likelihood method. (a) Distribution of cross-validation errors; (b) $\mathrm{K}=2$ and (c) $\mathrm{K}=3$, each vertical bar represents an individual, and the colours show the proportion of ancestry assigned to each of the clusters. 


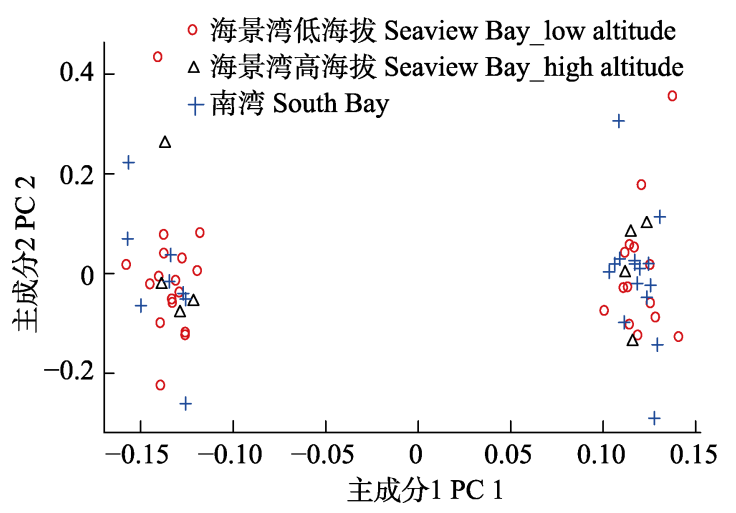

图3 基于62只阿德利企鹅110,451个SNPs的主成分分析 Fig. 3 PCA analysis based on 62 Adélie penguins using 110,451 SNPs

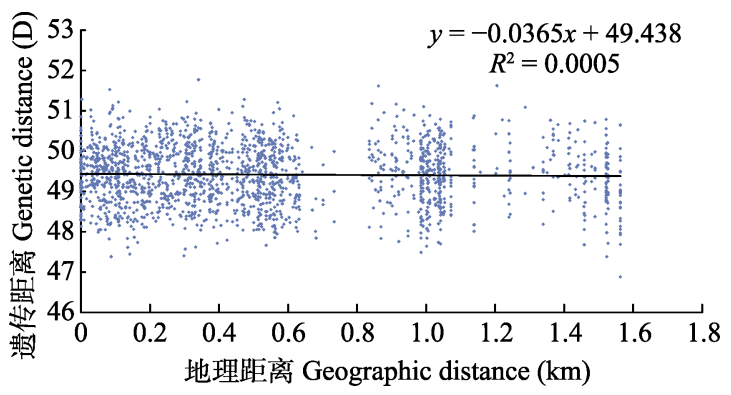

图4 阿德利企鹅个体间遗传距离与地理距离的Mantel检验 Fig.4 Mantel test between genetic distance and geographic distance among Adélie penguins

海拔区域、海景湾低海拔区域和南湾区域三个繁殖 种群之间未出现与地理分布格局相对应的遗传分 化(图3), 不存在显著的种群遗传结构。

\subsection{Mantel检验}

个体间的遗传距离与地理距离没有显著相关 性 $(P=0.400)$, 地理距离的增加并没有导致基因流 受限, 不存在距离隔离效应(图4)。

\section{3 讨论}

本研究对恩克斯堡岛上的阿德利企鹅进行全 基因组重测序, 利用贝叶斯法、最大似然法及主成分 分析等多种方法对分布于海景湾与南湾的阿德利企 鹅繁殖种群进行了遗传多样性调查和种群遗传结 构分析。结果表明, 海景湾与南湾附近分布的阿德 利企我没有显著的遗传差异, 海景湾附近的海拔差 异对阿德利企鹅的基因流与扩散也没有显著影响。

对于海景湾和南湾区域阿德利企鹅个体进行 遗传多样性分析发现, 阿德利企鹅核苷酸多态性水 平约为 $0.153 \%$ 。Younger等(2017)对南极8个帝企我鳥
(Aptenodytes forsteri)繁殖地个体进行遗传多样性分 析发现，帝企鹅的核苷酸多态性水平约为 $0.126 \%$, Clucas等(2016)对王企鹅(Aptenodytes patagonicus) 遗传多样性分析发现, 王企鹅的核苷酸多态性水平 约为 $0.122 \%$ 。相对帝企鹅和王企鹅来说，恩克斯堡 岛的阿德利企鹅具有较高的遗传多样性, 这可能与 本区域的繁殖种群持续时间较长, 且数量稳定, 保 留了较高的祖先遗传多样性有关。

Shepherd等(2005)对恩克斯堡岛上48只现生阿 德利企我的种群结构进行了研究, 他们通过 9 个微 卫星位点等位基因频率的分析发现，恩克斯堡岛的 现生种群倾向于随机交配 $(P=0.375)$, 可能不存在 明显的种群结构。但其研究中未结合采样个体的地 理信息对繁殖种群之间的遗传分化程度进行比较, 使用的少量微卫星位点数目对微地理尺度下种群 结构的分析也缺乏解析力, 采样过程中没有考虑海 拔是否会对阿德利企鹅群体间的基因流与扩散造 成影响。本研究应用全基因组水平上的SNPs数据对 种群遗传结构的分析结果更为精确, 为恩克斯堡岛 阿德利企鹅繁殖群体之间不存在显著的遗传分化 提供了充足的证据，同时也支持Shepherd等(2005) 通过标记观测所推测的, 本地区阿德利企鹅的归巢 行为并没有阻隔海景湾和南湾区域繁殖群体间的 基因交流。

本研究中主成分分析表明个体间存在一定程 度的分化，但个体间遗传分化情况与地理分布格局 无关。推测可能是祖先遗传多样性的保留，或者该 地区的繁殖种群来自两个曾经隔离的避难所。基于 现有数据无法确定遗传分化的来源, 有待于在更广 泛采集样品的基础上，进行演化历史的研究。

精确划分繁殖地边界对于制定精准的保护策 略具有重要意义。1994年对全球阿德利企鹅进行首 次普查时即提出了应当划分一个大的繁殖地或是 几个小繁殖地的保护管理问题, 并建议遵循以往数 目调查时的边界划分来定义阿德利企鹅繁殖地边 界(Lynch \& LaRue, 2016)。在本研究中, 利用全基因 组水平的SNPs位点进行分析, 表明恩克斯堡岛内 不同阿德利企我种群之间均不具有显著的遗传分 化，不同繁殖小群之间存在频繁的扩散，基因流较 强, 应属于同一个保护单元, 各繁殖小群不具有独 特的保护单元地位。因此, 可以将南湾和海景湾归 为同一个大繁殖地。而且, 海景湾的种群数量远远 
多于南湾，应作为重点保护种群，建议保护区划界 时涵盖海景湾繁殖种群的分布范围。

致谢: 感谢第34次南极科学考察队和罗斯海新站建 设队在项目执行中的大力支持, 感谢中国科学技术 大学高月嵩、黑龙江测绘地理信息局朱李忠在采样 和地图绘制中的帮助。

\section{参考文献}

Ainley DG, LeResche RE, Sladen WJL (1983) Breeding Biology of the Adélie Penguin. University of California Press, Oakland.

Alexander DH, Novembre J, Lange K (2009) Fast model-based estimation of ancestry in unrelated individuals. Genome Research, 19, 1655-1664.

Baroni C, Hall BL (2004) A new Holocene relative sea-level curve for Terra Nova Bay, Victoria Land, Antarctica. Journal of Quaternary Science, 19, 377-396.

Baroni C, Orombelli G (1991) Holocene raised beaches at Terra Nova Bay, Victoria Land, Antarctica. Quaternary Research, 36, 157-177.

Borowicz A, McDowall P, Youngflesh C, Sayre-McCord T, Clucas G, Herman R, Forrest S, Rider M, Schwaller M, Hart T, Jenouvrier S, Polito MJ, Singh H, Lynch HJ (2018) Multi-modal survey of Adélie penguin mega-colonies reveals the Danger Islands as a seabird hotspot. Scientific Reports, 8, 9.

Bromwich DH, Kurtz DD (1984) Katabatic wind forcing of the Terra Nova Bay polynya. Journal of Geophysical Research Oceans, 89, 3561-3572.

Clucas GV, Younger JL, Kao D, Emmerson L, Southwell C, Wienecke B, Rogers AD, Bost CA, Miller GD, Polito MJ, Lelliott P, Handley J, Crofts S, Phillips RA, Dunn MJ, Miller KJ, Hart T (2018) Comparative population genomics reveals key barriers to dispersal in Southern Ocean penguins. Molecular Ecology, 27, 4680-4697.

Clucas GV, Younger JL, Kao D, Rogers AD, Handley J, Miller GD, Jouventin P, Nolan P, Gharbi K, Miller KJ, Hart T (2016) Dispersal in the sub-Antarctic: King penguins show remarkably little population genetic differentiation across their range. BMC Evolutionary Biology, 16, 211.

Croxall JP, Prince PA (1979) Antarctic seabird and seal monitoring studies. Polar Record, 19, 573-595.

Danecek P, Auton A, Abecasis G, Albers CA, Banks E, DePristo MA, Handsaker RE, Lunter G, Marth GT, Sherry ST (2011) The variant call format and VCFtools. Bioinformatics, 27, 2156-2158.

Emslie SD, Coats L, Licht K (2007) A 45,000 yr record of Adélie penguins and climate change in the Ross Sea, Antarctica. Geology, 35, 61-64.
Freed D, Aldana R, Weber JA, Edwards JS (2017) The Sentieon Genomics Tools-A fast and accurate solution to variant calling from next-generation sequence data. bioRxiv, 115717.

Funk WC, McKay JK, Hohenlohe PA, Allendorf FW (2012) Harnessing genomics for delineating conservation units. Trends in Ecology \& Evolution, 27, 489-496.

Gorman KB, Talbot SL, Sonsthagen SA, Sage GK, Gravely MC, Fraser WR, Williams TD (2017) Population genetic structure and gene flow of Adélie penguins (Pygoscelis adeliae) breeding throughout the western Antarctic Peninsula. Antarctic Science, 29, 499-510.

Li H, Durbin R (2010) Fast and accurate long-read alignment with Burrows-Wheeler transform. Bioinformatics, 26, 589595.

Lischer HEL, Excoffier L (2012) PGDSpider: An automated data conversion tool for connecting population genetics and genomics programs. Bioinformatics, 28, 298-299.

Lorenzini S, Baroni C, Fallick AE, Baneschi I, Salvatore MC, Zanchetta G, Dallai L (2010) Stable isotopes reveal Holocene changes in the diet of Adélie penguins in Northern Victoria Land (Ross Sea, Antarctica). Oecologia, 164, 911-919.

Lynch HJ, LaRue MA (2016) First global census of the Adélie penguin. Auk, 133, 236.

Lyver POB, Barron M, Barton KJ, Ainley DG, Pollard A, Gordon S, McNeill S, Ballard G, Wilson PR (2014) Trends in the breeding population of Adélie penguins in the Ross Sea, 1981-2012: A coincidence of climate and resource extraction effects. PLoS ONE, 9, e91188.

Manel S, Schwartz MK, Luikart G, Taberlet P (2003) Landscape genetics: Combining landscape ecology and population genetics. Trends in Ecology \& Evolution, 18, 189-197.

Nielsen R, Paul JS, Albrechtsen A, Song Y (2011) Genotype and SNP calling from next-generation sequencing data. Nature Reviews Genetics, 12, 443-451.

Palsboll PJ, Berube M, Allendorf FW (2007) Identification of management units using population genetic data. Trends in Ecology \& Evolution, 22, 11-16.

Peakall R, Smouse PE (2012) GenAlEx 6.5: Genetic analysis in Excel. Population genetic software for teaching and research-an update. Bioinformatics, 28, 2537-2539.

Raj A, Stephens M, Pritchard JK (2014) fastSTRUCTURE: Variational inference of population structure in large SNP data sets. Genetics, 197, 573-589.

Ritchie PA, Millar CD, Gibb GC, Baroni C, Lambert DM (2004) Ancient DNA enables timing of the Pleistocene origin and Holocene expansion of two Adélie penguin lineages in Antarctica. Molecular Biology and Evolution, 21, 240-248.

Shepherd LD, Millar CD, Ballard G, Ainley DG, Wilson PR, Haynes GD, Baroni C, Lambert DM (2005) Microevolution and mega-icebergs in the Antarctic. Proceedings of the National Academy of Sciences, USA, 102, 16717-16722.

Stonehouse B (1969) Air census of two colonies of Adélie pen- 
guins (Pygoscelis adeliae) in Ross Dependency, Antarctica. Polar Record, 14, 471-475.

Walther GR, Post E, Convey P, Menzel A, Parmesan C, Beebee TJC, Fromentin JM, Hoegh-Guldberg O, Bairlein F (2002) Ecological responses to recent climate change. Nature, 416, 389-395.

Woehler EJ, Croxall JP (1997) The status and trends of Antarctic and sub-Antarctic seabirds. Marine Ornithology, 25, 43-66.

Younger JL, Clucas GV, Kao D, Rogers AD, Gharbi K, Hart T, Miller KJ (2017) The challenges of detecting subtle population structure and its importance for the conservation of emperor penguins. Molecular Ecology, 26, 3883-3897.

Zhang C, Dong SS, Xu JY, He WM, Yang TL (2019) PopL-
Ddecay: A fast and effective tool for linkage disequilibrium decay analysis based on variant call format files. Bioinformatics, 35, 1786-1788.

Zhang G, Li C, Li Q, Li B, Larkin DM, Lee C, Storz JF, Antunes A, Greenwold MJ, Meredith RW (2014) Comparative genomics reveals insights into avian genome evolution and adaptation. Science, 346, 1311-1320.

Zheng XW, Levine D, Shen J, Gogarten SM, Laurie C, Weir BS (2012) A high-performance computing toolset for relatedness and principal component analysis of SNP data. Bioinformatics, 28, 3326-3328.

(责任编委: 李明 责任编辑: 间文杰) 